\title{
PERCEPÇÃO DOS PROFISSIONAIS DE ENFERMAGEM FRENTE À PRATICA DA DISTANÁSIA E ORTOTANASIA
}

Ciro Félix Oneti¹, David Márcio de Oliveira Barreto², Elaine Lutz Martins²

Objetivo: analisar a produção científica acerca da percepção dos profissionais e estudantes de enfermagem diante da prática da distanásia e ortotanásia. Metodologia: realizou-se uma revisão integrativa de literatura, nas bases de dados Bireme, Medline, PubMed, com os descritores "enfermagem", "eutanásia”, “cuidados intensivos" e "percepções", nos idiomas português, inglês e espanhol, respectivamente. Foram encontrados 204 artigos, porém apenas 14 artigos responderam ao objetivo da pesquisa. A análise dos resultados ocorreu por meio da análise temática. Resultados: apontaram para a tomada de decisão do paciente e familiares; deficiência do ensino e na comunicação entre os profissionais sobre a temática; os sentimentos negativos vivenciados durante a assistência e medo de sofrer alguma sanção legal. Conclusão: é necessário investir mais na educação sobre o tema durante a graduação, estimular a comunicação entre os profissionais, além de valorizar seus sentimentos e preocupações.

Descritores: Enfermagem, Eutanásia, Direito de Morrer, Educação em Enfermagem, Percepção.

\section{PERCEPTION OF NURSING PROFESSIONALS BEFORE THE PRACTISE OF DISTHANASIAAND ORTHOTHANASIA}

Objective: To analyze the scientific production on the perception of professionals and nursing students with the practice of disthanasia and orthothanasia. Metodology: An integrative review of literature was realized in databases as: Bireme, Medline, PubMed with these keywords "nursing", "euthanasia", "intensive cares" and "perceptions" in the languages Portuguese, English and Spanish. 204 articles were found, but only 14 articles responded to the objective of the research. The analysis of the results was carried out through thematic analysis, grouped into thematic units. Results: they pointed to decision making process of the patient and his family: Lack of education and communication among professionals on the subject; Negative feelings experienced during service and fear of suffering any legal sanction. Conclusion: It is necessary to invest more in education on the subject during graduation, stimulate communication between professionals, and enhance their feelings and concerns.

Descriptors: Nursing, Euthanasia, Right to Die, Nursing Education, Perception.

\section{PERCEPCIÓN DE LOS PROFESIONALES DE ENFERMERÍA FRENTE A LA PRÁCTICA DE LA DISTANASIA Y ORTOTANASIA}

Objetivo: analizar la evidencia científica acerca de la percepción de los profesionales y estudiantes de enfermería en la práctica de la distanasia y ortotanasia. Metodología: Se llevó a cabo una revisión integradora de la literatura en las bases de datos Bireme, Medline, PubMed. Fuerón utilizados los descriptores "enfermeria”, “distanasia”, "vigilancia intensiva” y "percepciones" en los idiomas portugués, inglés e spañol. Fueron encontrados 204 artículos. Mas solamiente 14 artículos han respondido a el propósito de la investigación. El análisis se llevó a cabo mediante análisis temático, agrupados en unidades temáticas. Resultados: Han apuntado a la toma de decisiones del paciente y su familia; la educación de la discapacidad y la comunicación entre los profesionales en la materia; los sentimientos negativos experimentados durante el servicio y el miedo a sufrir de una sanción legal. Conclusión: es necesario invertir más en la educación sobre el tema durante la graduación, estimular la comunicación entre los profesionales, y mejorar sus sentimientos y preocupaciones.

Descriptores: Enfermería, Eutanasia, Direcho a Morir, Educacion en Enfermería, Percepción.

${ }^{1}$ Acadêmico de Enfermagem. Escola de Enfermagem da Universidade Federal do Amazonas - UFAM. Email: ciro_felixx@hotmail.com ${ }^{2}$ Enfermeiro. Mestre em Enfermagem. Escola de Enfermagem da UFAM. 


\section{INTRODUÇÃO}

Nos ambientes de cuidados em saúde, a ocorrência da morte é enfrentada com dificuldade e desafia os recursos tecnológicos de diversas áreas terapêuticas, por nem sempre serem capazes de curar, mas apenas prolongar o tempo de vida dos enfermos. Esse fato levanta questionamentos bioéticos, pois existe um declínio da qualidade de vida com certos investimentos que não conseguem recuperar o paciente ${ }^{(1)}$.

Essa realidade leva os profissionais de enfermagem a se questionarem "até que ponto a equipe multiprofissional deveria investir recursos assistenciais em determinado paciente?", ou "qual a melhor decisão: parar de investir recursos e permitir ao paciente uma morte natural ou prolongar sua vida a qualquer custo, mesmo que isso repercuta em mais sofrimento?". Tais questionamentos acabam convergindo para dois importantes dilemas éticos: a distanásia e a ortotanásia.

O termo Distanásia tem origem grega (dis: afastamento ou ato defeituoso e thanatos: morte) que se opõe à eutanásia por consistir no emprego de todos os meios terapêuticos possíveis, para atrasar ao máximo o momento da morte. Assim, o uso cada vez maior de tecnologia de ponta reflete no aumento da distanásia, tendo como cenário o contexto comercial-empresarial, no qual o procedimento terapêutico continua enquanto o paciente não morrer ou enquanto existir recursos para tal ${ }^{(2)}$.

Por outro lado, a ortotanásia, que também vem do grego, orto(certo) e thanatos(morte), significa para o paciente uma morte natural e com dignidade, no seu "tempo certo", pois trata-se de um processo que exclui as intervenções médicas, exceto aquelas que têm como finalidade amenizar a dor e o sofrimento(3)

Ressalta-se que no artigo 57 do Código de Ética Médica determina ser vedado ao médico "deixar de utilizar todos os meios disponíveis de diagnóstico e tratamento a seu alcance em favor da vida". Em contrapartida, o artigo 130 também veda ao médico "realizar experiências com novos tratamentos clínicos ou cirúrgicos em pacientes com afecção incurável ou terminal, sem que haja esperança razoável de utilidade para o mesmo, não the impondo sofrimentos adicionais"(4). Em outras palavras, proíbe ao médico tanto a omissão de socorro quanto à prática da distanásia.

Nesse contexto, percebe-se que os profissionais de enfermagem têm pouca participação no processo decisório, por não participarem das decisões médicas sobre os cuidados aos pacientes terminais ${ }^{(5)}$.

Analisando o Código de Ética dos Profissionais de Enfermagem, observa-se que este declara que "o profissional de enfermagem respeita a vida, a dignidade e os direitos humanos, em todas as suas dimensões" e proíbe, em seu artigo 29, ao profissional de enfermagem "promover a eutanásia ou participar em prática destinada a antecipar a morte do cliente"(6). Entretanto, o aludido diploma normativo da enfermagem não regulamenta as questões acerca da distanásia e ortotanásia.

Diariamente, os profissionais de enfermagem lidam com a morte das pessoas. Assim, dilemas éticos, que envolvem questões de distanásia e ortotanásia, trazem à tona a importância de se avaliar o que os profissionais de enfermagem pensam a respeito. Sendo assim, este estudo apresentou como objetivo analisar as evidências científicas sobre a percepção dos profissionais e estudantes de enfermagem diante da prática da distanásia e ortotanásia.

\section{METODOLOGIA}

Trata-se de uma revisão integrativa de literatura, que permite a sintese de múltiplos estudos publicados e possibilita conclusões gerais a respeito de uma particular área de estudo $^{7}$. Dessa forma, buscou-se investigar "qual a produção científica acerca do conhecimento dos profissionais de enfermagem sobre a distanásia e ortotanásia?".

A busca de dados ocorreu no período de novembro a dezembro de 2015, nas bases de dados Bireme, Medline, PubMed e nas bibliotecas virtual Scielo e Cochrane. Como descritores utilizou-se "enfermagem", “eutanásia”, “cuidados intensivos" e "percepções", além das combinações no idioma inglês e espanhol.

Inicialmente, foram encontrados 204 artigos, os mesmos foram analisados individualmente, conforme critérios de inclusão: estudos publicados a partir de 2001; disponibilidade de texto na íntegra; publicados nos idiomas português, inglês ou espanhol; trabalhos originais; e que respondessem à questão de pesquisa. Assim, após leitura exaustiva, realizada em pares, foram encontrados 14 artigos que atenderam aos critérios de inclusão. Os mesmos foram classificados em códigos para melhor compreensão dos resultados. Com a análise textual discursiva foi possivel agrupar os resultados em núcleos temáticos e discutir com literatura especializada.

\section{RESULTADOS}

Dos 14 trabalhos incluídos no estudo, todos se encontram publicados em língua portuguesa e em periódicos brasileiros. Dentre eles, um (7,1\%) foi publicado entre os anos 2001 a 2005 , cinco (35,7\%) foram publicados entre 2006 a 2010, e oito $(57,1 \%)$ foram publicados entre os anos de 2011 a 2015. Dessa forma, observou-se que o tema vem ganhando importância no âmbito da pesquisa científica.

Para melhor entendimento do leitor e discussão dos resultados, organizamos a Tabela 1, que apresenta o código de classificação dos artigos selecionados, conforme o ano de publicação, referência, título e tipo de pesquisa. 
Tabela 1: Artigos incluidos na revisão integrativa de literatura, segundo código, ano, referência, título do artigo e tipo de pesquisa.

\begin{tabular}{|c|c|c|c|c|}
\hline Código & Ano & & Titulo & Tipo de pesquisa \\
\hline $\mathrm{Al}$ & 2005 & $\begin{array}{l}\text { Toffoletto, M.C.; et al. } \\
\text { Acta paul. enferm.;18(3): 307-12. }\end{array}$ & $\begin{array}{l}\text { A distanásia como geradora de dilemas } \\
\text { éticos nas Unidades de Terapia Intensiva: } \\
\text { considerações sobre a participação dos } \\
\text { enfermeiros }\end{array}$ & $\begin{array}{l}\text { Revisão integrativa } \\
\text { de literatura }\end{array}$ \\
\hline $\mathrm{A} 2$ & 2008 & $\begin{array}{l}\text { Balla, A.; Brasil, R.; Hass, E. } \\
\text { Bioethikos. - Centro Universitário } \\
\text { São Camilo;2(2):204-213 }\end{array}$ & $\begin{array}{l}\text { Percepção do enfermeiro em relação à } \\
\text { ortotanásia }\end{array}$ & Qualitativa \\
\hline A3 & 2009 & $\begin{array}{l}\text { Menezes, M.B.; Selli, L. } \\
\text { Rev. Latino-Am. Enfermagem; 17(4) }\end{array}$ & $\begin{array}{l}\text { Distanásia: percepção dos profissionais da } \\
\text { enfermagem }\end{array}$ & Qualitativa \\
\hline A4 & 2009 & $\begin{array}{l}\text { Silva, F.S.; Pachemshy, L.R.; } \\
\text { Rodrigues, I.G. } \\
\text { Rev. bras. ter. intensiva; 12(2): 148- } \\
154\end{array}$ & $\begin{array}{l}\text { Percepção de enfermeiros intensivistas } \\
\text { sobre distanásia em Unidade de Terapia } \\
\text { Intensiva }\end{array}$ & Qualitativa \\
\hline A5 & 2009 & $\begin{array}{l}\text { Santana, J.C.B.;et al. } \\
\text { Bioethikos-Centro Universitário } \\
\text { São Camilo;3(1):77-86 }\end{array}$ & $\begin{array}{l}\text { Cuidados paliativos aos pacientes } \\
\text { terminais: percepção da equipe de } \\
\text { enfermagem }\end{array}$ & Qualitativa \\
\hline A6 & 2010 & $\begin{array}{l}\text { Bisogno, S.B.B.C.; Quintana, A.M.; } \\
\text { Camargo, V.P. } \\
\text { REME - Rev. Min. Enferm.;14(3): } \\
\text { 327-334 }\end{array}$ & $\begin{array}{l}\text { Entre a vida enferma e a morte sadia: a } \\
\text { ortotanásia na vivência de enfermeiros em } \\
\text { Unidade de Terapia Intensiva }\end{array}$ & Qualitativa \\
\hline A7 & 2012 & $\begin{array}{l}\text { Vasques, T.C.S; et al. } \\
\text { Rev. Eletr. Enf. } \\
\text { [Internet];15(3):772-9 }\end{array}$ & $\begin{array}{l}\text { Percepção dos trabalhadores de } \\
\text { enfermagem acerca dos cuidados } \\
\text { paliativos e de sua implementação }\end{array}$ & Qualitativa \\
\hline A8 & 2013 & $\begin{array}{l}\text { Santos, LRG; Menezes, MP; } \\
\text { Mayumiobanagradvohl, S. } \\
\text { Ciência \& Saúde Coletiva; 18(9): } \\
\text { 2645-2651 }\end{array}$ & $\begin{array}{l}\text { Conhecimento, envolvimento e } \\
\text { sentimentos de concluintes dos cursos de } \\
\text { medicina, enfermagem e psicologia sobre } \\
\text { ortotanásia }\end{array}$ & Qualitativa \\
\hline A9 & 2013 & $\begin{array}{l}\text { Santana, J.C.B.; et al. } \\
\text { Rev. Bioét.; 12(2): 298-307 }\end{array}$ & $\begin{array}{l}\text { Docentes de enfermagem e } \\
\text { terminalidade em condições dignas }\end{array}$ & Qualitativa \\
\hline Alo & 2013 & $\begin{array}{l}\text { Brasil, N.M.; Oliveira, A.K.; } \\
\text { Rocha, L. } \\
\text { InterScientia; 1(3):93-102 }\end{array}$ & $\begin{array}{l}\text { Distanásia: a percepção do enfermeiro } \\
\text { quanto a sua prática em UTI }\end{array}$ & Qualitativa \\
\hline All & 2013 & $\begin{array}{l}\text { Felix, Z.C.; et al } \\
\text { Ciênc. Saúde Coletiva; 18(9): } \\
\text { 2733-2746 }\end{array}$ & $\begin{array}{l}\text { Eutanásia, distanásia e ortotanásia: } \\
\text { revisão integrativa de literatura }\end{array}$ & $\begin{array}{l}\text { Revisão integrativa } \\
\text { de literatura }\end{array}$ \\
\hline $\mathrm{Al} 2$ & 2014 & $\begin{array}{l}\text { Fernandes, A.S.; Brasil, S.; } \\
\text { Coelho, P.F. } \\
\text { Revista CUIDARTE; 5(2): 813-9 }\end{array}$ & $\begin{array}{l}\text { Distanásia em unidade de cuidados inten- } \\
\text { sivos e a visão de enfermagem: revisão } \\
\text { integrativa }\end{array}$ & $\begin{array}{l}\text { Revisão integrativa } \\
\text { de literatura }\end{array}$ \\
\hline $\mathrm{Al3}$ & 2015 & $\begin{array}{l}\text { Matos, MC; Rosa, DOS; Anjos, K.F. } \\
\text { Rev Rene; 16(6):817-25. }\end{array}$ & $\begin{array}{l}\text { Conhecimentos de estudantes de } \\
\text { enfermagem sobre distanásia }\end{array}$ & Qualitativa \\
\hline $\mathrm{Al} 4$ & 2015 & $\begin{array}{l}\text { Santana, J.C.B.; et al. } \\
\text { Enfermagem Revista;18(2): 29-41. }\end{array}$ & $\begin{array}{l}\text { Vivências do cuidar de pacientes na } \\
\text { terminalidade da vida: percepção de um } \\
\text { grupo de acadêmicos de enfermagem }\end{array}$ & Qualitativa \\
\hline
\end{tabular}


Ainda, a análise dos resultados permitiu identificar núcleos temáticos, como: "tomada de decisão", "deficiência no ensino e na comunicação entre os profissionais sobre a temática", "sentimentos negativos vivenciados durante a assistência e medo de sofrer alguma sanção legal", explanados e discutidos a seguir.

\section{DISCUSSÃO}

Tomada de decisão

Um artigo (Al2) evidenciou a importância da opinião do paciente na decisão sobre como a equipe deve proceder durante o seu estado terminal. Uma percepção sensata, pois indica a valorização da opinião de quem recebe os cuidados, demonstrando respeito e humanização.

Sabe-se que o modelo assistencial prioriza o cuidado de forma humanizada e integral, reconhecendo que o paciente tem o direito de manifestar sua vontade com antecipação quanto ao tratamento que irá receber, ou de simplesmente recusar ser tratado, pois preconiza o cuidado individualizado(8).

Esse aspecto nos faz refletir sobre a autonomia dos pacientes durante a internação. Também é preocupante se seus desejos ou de seus familiares estão sendo considerados pela equipe que os atende, no que tange ao tratamento oferecido.

Refletindo sobre os aspectos bioéticos em saúde na tomada de decisão, percebe-se que o princípio ético da beneficência acaba ficando em segundo plano quando são utilizados tratamentos agressivos e sem garantia de sucesso nos pacientes terminais. Ainda, o princípio da autonomia, que pertence a todos os adultos em bom estado de consciência, parece também ser oculto, quando se adotam condutas terapêuticas diante da impossibilidade de comunicação do paciente ${ }^{(9)}$. Infelizmente, pouco se sabe sobre a autonomia desses pacientes acerca da tomada de decisões em cuidados paliativos $^{(8)}$

Referente à participação da família na tomada de decisão, um artigo (A8) expressou que a equipe de saúde valoriza a opinião do familiar quanto ao que será decidido sobre a terapêutica ideal, o que é muito positivo, pois demonstra que está sendo respeitada a autonomia da família e abertura para o diálogo e escuta ativa.

Corroborando com achados deste estudo, sabe-se que a participação da família ocorre em casos de supressão da capacidade de comunicação do paciente, assim, os familiares assumem a responsabilidade de decidir pelo futuro ou de comunicar suas decisões prévias ${ }^{(8)}$.

Deficiência no ensino e na comunicação entre os profissionais sobre a temática

Um aspecto preocupante deste estudo é o fato de algumas instituições de ensino superior não abordarem com profundidade essa temática no ensino da bioética durante a graduação. Oito artigos (Al, A2, A3, A6, A7, A8, Al3, Al4) apontaram para essa deficiência no conhecimento. Além disso, acadêmicos revelaram desconhecer ou conhecer parcialmente o significado de termos como distanásia e ortotanásia, conforme os estudos evidenciaram. Certos profissionais, que já atuam há anos, também revelaram ter pouco domínio sobre esses termos e/ou pouca abordagem sobre bioética quando ainda eram graduandos.

Acredita-se que a carência na preparação bioética do profissional de enfermagem certamente é um dos principais fatores que proporcionam situações de desgaste emocional e despreparo para a tomada de decisões conjuntas, sobre como delinear a abordagem terapêutica de certos pacientes ${ }^{(10)}$

Nesse sentindo, seis estudos (Al, A3, A4, A6, A7, All) evidenciaram a necessidade de educação continuada nos ambientes de trabalho para atualizar os profissionais de saúde. Sabe-se que a educação continuada consiste em aporte ou extensão do que foi ensinado na graduação, sendo desenvolvido no local de trabalho do profissional(11)

Outra evidência foi a necessidade de mais comunicação entre os profissionais, a qual apenas três artigos (Al, A4, Al0) abordaram a comunicação dentro do ambiente de cuidados intensivos. Esses estudos indicaram que a falta de comunicação faz com que profissionais de diferentes turnos tomem condutas divergentes do que foi estabelecido no turno anterior, prejudicando muitas vezes o cuidado integral ao paciente.

Sentimentos negativos vivenciados durante a assistência e medo de sofrer alguma sanção legal

Outro elemento muito presente nos estudos investigados foram os sentimentos de fragilidade e angústia, por parte da equipe de enfermagem. Assim, seis artigos (Al, A7, A8, A9, $\mathrm{AlO}, \mathrm{Al4}$ ) revelaram que o enfermeiro acaba experimentando sentimentos angustiantes dentro de ambientes de alta complexidade, pois tais profissionais tendem a considerar a morte como um fracasso, uma derrota ou falha na qualidade de sua assistência.

Os sentimentos mais aflitivos se mostram em momentos que se presencia a iminência da morte dos pacientes mais jovens. Tem sido verificado que o sofrimento e a angústia produzidos no ambiente de trabalho acabam sendo levados para a casa ou que perduram por mais tempo ${ }^{(12)}$

Fortalecer a espiritualidade (A14) foi indicada como uma estratégia pelos enfermeiros, visando diminuir o impacto emocional causado por presenciar mortes em seu ambiente de trabalho. Nesse sentido, associado com a espiritualidade, técnicas de enfrentamento emocional e social poderão auxiliar na diminuição da frustação, pois a perda de um 
paciente pode representar um fracasso biomédico(10). Já para o paciente, o apoio religioso apresenta mais resistência para enfrentar o advento da morte, serenidade e bem-estar para passar seus últimos momentos ${ }^{(13)}$.

Ainda, os sentimentos de angústia verbalizados pelos enfermeiros, muitas vezes, é devido à incerteza sobre qual é a melhor ação para um paciente sem chances de recuperação. Nesse sentido, um artigo (A6) evidenciou que os profissionais de enfermagem temem receber punições legais, por conta de atos cometidos no seu serviço envolvendo os ambientes de alta complexidade. A divergência de opiniões entre a equipe multiprofissional os levam a ficar inibidos de manifestar opiniões. No entanto, cabe ao profissional médico decidir sobre atos como reanimar ou não um certo paciente, conforme o prognóstico do mesmo.

Acredita-se que essa problemática presente nos serviços de saúde é devido à falta de atualizações de conhecimentos de alguns dos membros da equipe e também por deficiências de comunicação, pois existe um grande receio de sofrer punições legais por seus atos dentro de ambientes de alta complexidade e no cuidado aos pacientes terminais.

\section{CONCLUSÃO}

Após a realização desta revisão integrativa de literatura constatou-se aumento de publicações nos últimos anos no Brasil, porém a temática apresenta-se limitada no que tange à formação universitária e à atuação dos profissionais na assistência de enfermagem.

Os resultados encontrados apontam para a necessidade de se fortalecer a prática de incluir o paciente e seus familiares no processo de tomada de decisão quanto ao tratamento, incentivar maior comunicação e troca de experiências entre os membros da equipe de saúde, reconhecer a existência de fragilidades emocionais dos profissionais e o receio que sentem de sofrer represálias ou sanções durante o exercício da função.

Ainda, identificou-se a necessidade de discussão e reflexão sobre a formação dos profissionais de enfermagem e a introdução dessa temática nos currículos acadêmicos de ensino médio e superior, visando a formação de profissionais capacitados para atuarem nessa fase do fim da vida de forma humana, obedecendo os princípios da bioética.

\section{REFERÊNCIAS}

1. Félix ZC, Costa SFG, Alves AMPM, Andrade CG, Duarte MCS, Brito FM. Eutanásia, distanásia e ortotanásia: revisão integrativa de literatura. Ciênc Saúde Coletiva. 2013; 18(9): 2733-46.

2. Feio AGO, Oliveira CC. Responsabilidade e tecnologia: a questão da distanásia. Rev. Bioética (impress). 2011; 19(3): 615-30.

3. Araguaia M. Ortotanásia. Brasil Escola [homepage na internet]. 2015. Disponivel em: http://www.brasilescola.com/sociologia/ortotanasia. htm. Acesso em: 23/011/2015.

4. Brasil. Código de Ética Médica e Normas Complementares. São Paulo: Edipro;2010

5. Menezes MB, Selli L, Alves JS. Distanásia: percepção dos profissionais da enfermagem. Rev. Latino-Am. Enfermagem. 2009; 17 (4): 443-48.

6. Lopes ND, Almeida CS, Barreto DMO. Conselho Regional de Enfermagem do Amazonas. Legislação dos Profissionais de Enfermagem. Manaus: Vitória: 2014.

7. Mendes KDS, Silveira RCCP, Galvão CM. Revisão integrativa: método de pesquisa para a incorporação de evidências na saúde e na enfermagem. Texto Contexto Enferm. 2008; 17(4): 758-64.
8. Vieira RW, Goldim JR. Bioética e cuidados paliativos: tomada de decisões e qualidade de vida. Acta Paul Enferm. 2012; 25(3): 334-9.

9. Clotet J. Reconhecimento e institucionalização da autonomia do paciente: um estudo da the pacient self-determination act. Revista bioética. 2009: 1(2): 157-63.

10. Almeida AS, Barle ELD, Tomaschewski-Barlem JG, Lunardi VL. Produção cientifica da enfermagem sobre morte/morrer em unidades de terapia intensiva. Rev. Enfermagem em foco. 2013:4 (3,4) 179-183.

11. Montanha D, Peduzzi M. Educação permanente em enfermagem levantamento de necessidades e resultados esperados segundo a concepção dos trabalhadores. Rev. Esc. Enferm. USP. 2010; 44(3): 597604 .

12. Martins JT, Robazzi MLCC. O trabalho do enfermeiro em unidade de terapia intensiva: sentimentos de sofrimento. Rev. Latino-Am Enfermagem. 2009: 17(1): 52-58.

13. Brito FM, Costa ICP, Andrade CG, Lima KFO, Costa SFG, Lopes MEL. Espiritualidade na iminência da morte: estratégia para humanizar o cuidar em enfermagem. Rev. Enferm. UERJ. 2013; 21(4): 483-9. 